PROCEEDINGS OF THE

AMERICAN MATHEMATICAL SOCIETY

Volume 135, Number 9, September 2007, Pages 2769-2777

S 0002-9939(07)08928-9

Article electronically published on February 9, 2007

\title{
UNBOUNDED SOLUTIONS AND PERIODIC SOLUTIONS FOR SECOND ORDER DIFFERENTIAL EQUATIONS WITH ASYMMETRIC NONLINEARITY
}

\author{
XIONG LI AND ZIHENG ZHANG \\ (Communicated by Carmen C. Chicone)
}

\begin{abstract}
In this paper we will prove the coexistence of unbounded solutions and periodic solutions for the asymmetric oscillator$$
\ddot{x}+f(\dot{x})+a x^{+}-b x^{-}=\varphi(t, x),
$$

where $a$ and $b$ are positive constants satisfying the nonresonant condition

$$
\frac{1}{\sqrt{a}}+\frac{1}{\sqrt{b}} \notin \mathbb{Q}
$$

and $\varphi(t, x)$ is $2 \pi$-periodic in the first variable and bounded.
\end{abstract}

\section{INTRODUCTION}

Let us consider a particle of mass $m=1$ attached to two springs with Hooke's constants $\omega_{1}^{2}$ and $\omega_{2}^{2}$. The second spring is only on one side and has a stop at the origin. Under the action of an external forcing $p=p(t)$, the equation of motion is

$$
\ddot{x}+a x^{+}-b x^{-}=p(t),
$$

where $x^{+}=\max (x, 0), x^{-}=\max (-x, 0)$, and $a=\omega_{1}^{2}, b=\omega_{1}^{2}+\omega_{2}^{2}$. The above equation will be referred to as the equation of the asymmetric oscillator, or the equation with 'jumping nonlinearity'. Also this equation appeared as a simplified version of the model of the suspension bridge of Lazer and McKenna [13].

The same equation had been studied by Fučik [8] and Dancer 4 in their investigation of boundary value problems. After these works, the Dirichlet, Neumann and periodic problems for (1.1) have been the subject of several papers [9, 10, 12 and the references therein.

Now let us review some results on the periodic problem for (1.1). They can be seen as a sort of Fredholm Alternative for the asymmetric oscillator. In the space of parameters $(a, b) \in \mathbb{R}_{+}^{2}$, define the family of curves

$$
\mathcal{C}_{n}: \frac{1}{\sqrt{a}}+\frac{1}{\sqrt{b}}=\frac{2}{n}, \quad n \in \mathbb{N} .
$$

Received by the editors May 4, 2006.

2000 Mathematics Subject Classification. Primary 34C11, 34C25.

Key words and phrases. Unbounded solutions, periodic solutions, asymmetric oscillator.

This project was supported by the Program for New Century Excellent Talents of Ministry of Education of China and the National Natural Science Foundation of China (Grant No. 10671020 and 10301006).

(C)2007 American Mathematical Society Reverts to public domain 28 years from publication 
These curves are similar to hyperbolae, and the intersection of each of them with the diagonal $a=b$ is the point $\left(n^{2}, n^{2}\right)$. These points are in the periodic spectrum of harmonic oscillators. The role played by the set $\left\{n^{2}: n \in \mathbb{N}\right\}$ in the periodic linear problem is now played by $\Sigma=\bigcup_{n=1}^{\infty} \mathcal{C}_{n}$ in the asymmetric oscillator. Namely, if $(a, b) \notin \Sigma$, then Eq.(1.1) has a $2 \pi$-periodic solution for each $f \in L^{1}(\mathbb{R} / 2 \pi \mathbb{Z})$; if $(a, b) \in \Sigma$, then there exists a function $f \in L^{1}(\mathbb{R} / 2 \pi \mathbb{Z})$ such that Eq.(1.1) has no $2 \pi$-periodic solutions. We refer to [3, 6], 13] for more details.

Recently, the bounded or unbounded motions for the asymmetric oscillator (1.1) was considered by Ortega [1, [17, [19] and Liu [16]. The existence of bounded or unbounded solutions for the asymmetric oscillator (1.1) with the resonant case $\frac{1}{\sqrt{a}}+\frac{1}{\sqrt{b}}=2 \frac{m}{n} \in \mathbb{Q}$ essentially depends on the oscillatory property of the function $\Phi_{p}(\theta)$ which relates to the autonomous system

$$
\ddot{x}+a x^{+}-b x^{-}=0
$$

and the $2 \pi$-periodic external forcing $p$, and which is given by

$$
\Phi_{p}(\theta)=\int_{0}^{2 \pi} p(m t) C(\theta+m t) d t, \quad \theta \in \mathbb{R},
$$

where $C(t)$ is the solution of $(1.2)$ with the initial condition $x(0)=1, \dot{x}(0)=0$. That is, if the function $\Phi_{p}(\theta)$ has no zeros, all solutions of (1.1) are bounded; if all zeros of $\Phi_{p}(\theta)$ are nondegenerate, all solutions of (1.1) with large initial conditions are unbounded. Moreover, when $(a, b) \notin \Sigma$, the results of Dancer imply the existence of a periodic solution. In consequence, there exists a function $f \in L^{1}(\mathbb{R} / 2 \pi \mathbb{Z})$ such that Eq.(1.1) has unbounded solutions, and also periodic solutions. This is a genuine nonlinear phenomenon. In the nonresonant case $\frac{1}{\sqrt{a}}+\frac{1}{\sqrt{b}} \notin \mathbb{Q}$, the condition $\int_{0}^{2 \pi} p(t) d t \neq 0$ guarantees the boundedness of (1.1); see [19].

More recently, the problem of boundedness of all solutions for the following nonconservative system

$$
\ddot{x}+f(x) \dot{x}+n^{2} x+g(x)=p(t)
$$

had been studied in [11] with $n \in \mathbb{N}$. In [20], Wang had studied the unboundedness of solutions for

$$
\ddot{x}+f(x) \dot{x}+a x^{+}-b x^{-}=p(t)
$$

in the nonresonant case. In [15], the first author had investigated the problem of boundedness and unboundedness of all solutions for the nonlinear oscillator

$$
\ddot{x}+f(\dot{x})+a x^{+}-b x^{-}+g(x)=p(t)
$$

in the resonant case. The boundedness of all solutions for Eq.(1.3) in the nonresonant case was discussed in another paper [14.

In this paper we study the coexistence of unbounded solutions and periodic solutions for the asymmetric oscillator

$$
\ddot{x}+f(\dot{x})+a x^{+}-b x^{-}=\varphi(t, x)
$$

under the nonresonant condition

$$
\frac{1}{\sqrt{a}}+\frac{1}{\sqrt{b}} \notin \mathbb{Q}
$$

and $\varphi(t, x)$ is $2 \pi$-periodic in the first variable and bounded. 
We suppose that

(H1) $\lim _{x \rightarrow+\infty} f(x):=f(+\infty) \neq \lim _{x \rightarrow-\infty} f(x):=f(-\infty)$.

(H2) $\lim _{x \rightarrow+\infty} \varphi(t, x):=\varphi(t,+\infty)$ and $\lim _{x \rightarrow-\infty} \varphi(t, x):=\varphi(t,-\infty)$ exist uniformly in $t$.

Now we are ready to state our main results.

Theorem 1.1. Under the nonresonant condition (1.5) and (H1)-(H2), there exists $R_{0}>0$ such that every solution $x(t)$ of $E q .(1.4)$ satisfying for some $t_{0} \in \mathbb{R}$

$$
x\left(t_{0}\right)^{2}+\dot{x}\left(t_{0}\right)^{2}>R_{0}^{2}
$$

are unbounded. Moreover, Eq.(1.4) has at least one $2 \pi$-periodic solution.

We remark that Theorem 1.1 can be applied to Eq.(1.3). For example, all solutions of the equation

$$
\ddot{x}+\arctan \dot{x}+x^{+}-2 x^{-}+\frac{1}{1+x^{2}}=\cos t
$$

with large initial conditions are unbounded. We also point out that in order to obtain the boundedness of all solutions for Eq.(1.3), we have to assume that $f$ is even, which implies that $f(+\infty)=f(-\infty)$; see Theorem 1.1 in [14. For example, all solutions of the equation

$$
\ddot{x}+(\arctan \dot{x})^{2}+x^{+}-2 x^{-}+\frac{1}{1+x^{2}}=\cos t
$$

are bounded. Therefore, the assumption $f(+\infty)=f(-\infty)$ is sharp in determining the boundedness of all solutions for Eq.(1.3).

The rest of the paper is organized as follows. In Section 2 we deal with unbounded motions of the planar mappings and give the proof of Theorem 1.1 in Section 3.

\section{UnBounded MOTIONS OF THE PLANAR MAPPING}

The proof of our main result will be a consequence of the application of the abstract results due to Wang [20, concerning the dynamics of a class of planar mappings. In order to state these propositions, we need some preliminaries.

For any $\sigma>0$, let us define

$$
E_{\sigma}=\{(\theta, r) \in \mathbb{R} \times(0,+\infty): r \geq \sigma\} .
$$

Moreover, we assume that $P: E_{\sigma} \rightarrow \mathbb{R}^{2}$ is a one-to-one and continuous mapping of the form

$$
\left\{\begin{array}{l}
\theta_{1}=\theta+2 \alpha \pi+\frac{1}{r} \mu_{1}(\theta)+H(\theta, r), \\
r_{1}=r+\mu_{2}(\theta)+G(\theta, r),
\end{array}\right.
$$

where

(2.2) $\quad \alpha \in \mathbb{R}^{+} \backslash \mathbb{Q}, \mu_{1}, \mu_{2}: \mathbb{S}^{1} \rightarrow \mathbb{S}^{1}$ are Lipschitz continuous $\left(\mathbb{S}^{1}=\mathbb{R} / 2 \pi \mathbb{Z}\right)$,

$H$ and $G$ are continuous functions, which are $2 \pi$-periodic in $\theta$ and satisfy

$$
r|H(\theta, r)|+|G(\theta, r)| \rightarrow 0
$$

uniformly with respect to $\theta \in \mathbb{R}$ as $r \rightarrow+\infty$.

Let us take $\left(\theta_{0}, r_{0}\right) \in E_{\sigma}$, and denote by $\left\{\left(\theta_{n}, r_{n}\right)\right\}$ the orbit of the mapping $P$ through the point $\left(\theta_{0}, r_{0}\right)$. That is to say,

$$
\left(\theta_{n+1}, r_{n+1}\right)=P\left(\theta_{n}, r_{n}\right) \text {. }
$$


The following abstract results taken from [20] will be applied to prove our main theorem.

Proposition 2.1. Assume that the conditions (2.2), (2.3) hold and

$$
\int_{0}^{2 \pi} \mu_{2}(\theta) d \theta>0 .
$$

Then there exists $R_{0}>\sigma$ such that if $r_{0}>R_{0}$, the orbit $\left\{\left(\theta_{n}, r_{n}\right)\right\}$ satisfies

$$
\lim _{n \rightarrow+\infty} r_{n}=+\infty \text {. }
$$

Proposition 2.2. Assume that the conditions (2.2), (2.3) hold and

$$
\int_{0}^{2 \pi} \mu_{2}(\theta) d \theta<0 .
$$

Then there exists $R_{0}>\sigma$ such that if $r_{0}>R_{0}$, the orbit $\left\{\left(\theta_{n}, r_{n}\right)\right\}$ satisfies

$$
\lim _{n \rightarrow-\infty} r_{n}=+\infty \text {. }
$$

\section{THE PROOF OF THE MAIN RESUlT}

In this section, at first we introduce action-angle variables for Eq.(1.4). Secondly, we will give an expression for the Poincaré map of Eq.(1.4) under action-angle variables. Finally the proof of Theorem 1.1 will be given.

3.1. Action-angle variables. In order to apply the propositions we will take $P$ as the Poincaré map associated to Eq.(1.4). Therefore, some asymptotic estimates on this map will be needed. To this end, first we consider the piecewise linear equation

$$
\ddot{x}+a x^{+}-b x^{-}=0,
$$

and denote by $C(t)$ the solution satisfying the initial condition

$$
x(0)=1, \quad \dot{x}(0)=0 .
$$

It is easy to see that $C(t)$ is a $\tau$-periodic function with $\tau=\frac{\pi}{\sqrt{a}}+\frac{\pi}{\sqrt{b}}$ which is given by

$$
C(t)= \begin{cases}\cos \sqrt{a} t, & 0 \leq|t| \leq \frac{\pi}{2 \sqrt{a}}, \\ -\sqrt{\frac{a}{b}} \sin \sqrt{b}\left(t-\frac{\pi}{2 \sqrt{a}}\right), & \frac{\pi}{2 \sqrt{a}} \leq|t| \leq \frac{\tau}{2} .\end{cases}
$$

The derivative of $C(t)$ will be denoted by $S(t)=\dot{C}(t)$. Obviously $C(t)$ and $S(t)$ satisfy the following properties:

(i) $C(t+\tau)=C(t), S(t+\tau)=S(t)$ and $C(0)=1, S(0)=0$.

(ii) $C(t) \in C^{2}(\mathbb{R}), S(t) \in C^{1}(\mathbb{R})$.

(iii) $\dot{C}(t)=S(t), \dot{S}(t)=-\left(a C^{+}(t)-b C^{-}(t)\right)$.

(iv) $S(t)^{2}+a C^{+}(t)^{2}+b C^{-}(t)^{2}=a, \forall t \in \mathbb{R}$.

We introduce the canonical change of variables

$$
x=\gamma I^{\frac{1}{2}} C\left(\frac{\theta}{\omega}\right), \quad y=\gamma I^{\frac{1}{2}} S\left(\frac{\theta}{\omega}\right),
$$

where $\omega=\frac{2 \pi}{\tau}$ and $\gamma=\sqrt{\frac{2 \omega}{a}}$ with $I>0$ and $\theta \in \mathbb{R}$. We observe that the pair $(\theta, I)$ represents the action-angle variables associated to the unperturbed equation $\ddot{x}+a x^{+}-b x^{-}=0$. 
3.2. An expression for the Poincaré map of (1.4). We only deal with the case $f(+\infty)<f(-\infty)$; the case $f(-\infty)<f(+\infty)$ is completely analogous. Without loss of generality we may assume that $f(+\infty)<0<f(-\infty)$. In fact, taking some constant $d$ satisfying $f(+\infty)<d<f(-\infty)$, we consider the following equation:

$$
\ddot{x}+f(\dot{x})-d+a x^{+}-b x^{-}=\varphi(t, x)-d,
$$

which is just Eq.(1.4). Set

$$
\bar{f}(x)=f(x)-d, \quad \bar{\varphi}(t, x)=\varphi(t, x)-d .
$$

Then $\bar{f}(x)$ and $\bar{\varphi}(t, x)$ still satisfy all the conditions in the Theorem 1.1, and $\bar{f}(+\infty)<0<\bar{f}(-\infty)$.

Now consider the equivalent system of Eq.(3.1)

$$
\dot{x}=y, \quad \dot{y}=-\left(a x^{+}-b x^{-}\right)-f(y)+\varphi(t, x) .
$$

Under action-angle transformation, Eq.(3.1) becomes

$$
\left\{\begin{array}{l}
\frac{d \theta}{d t}=\omega+\frac{\gamma}{2} I^{-\frac{1}{2}} f\left(\gamma I^{\frac{1}{2}} S\left(\frac{\theta}{\omega}\right)\right) C\left(\frac{\theta}{\omega}\right)-\frac{\gamma}{2} I^{-\frac{1}{2}} \varphi\left(t, \gamma I^{\frac{1}{2}} C\left(\frac{\theta}{\omega}\right)\right) C\left(\frac{\theta}{\omega}\right), \\
\frac{d I}{d t}=-\frac{2}{a \gamma} I^{\frac{1}{2}} f\left(\gamma I^{\frac{1}{2}} S\left(\frac{\theta}{\omega}\right)\right) S\left(\frac{\theta}{\omega}\right)+\frac{2}{a \gamma} I^{\frac{1}{2}} \varphi\left(t, \gamma I^{\frac{1}{2}} C\left(\frac{\theta}{\omega}\right)\right) S\left(\frac{\theta}{\omega}\right) .
\end{array}\right.
$$

Denote by $\left(\theta\left(t ; \theta_{0}, I_{0}\right), I\left(t ; \theta_{0}, I_{0}\right)\right)$ the solution of (3.2) satisfying the initial value $\theta(0)=\theta_{0}, I(0)=I_{0}$. By the assumption that $f(x)$ and $\varphi(t, x)$ are bounded, for large values of $I_{0}$, this solution is defined for all $t \in[0,2 \pi]$. Thus we can define the Poincaré mapping

$$
\theta_{1}=\theta\left(2 \pi ; \theta_{0}, I_{0}\right), \quad I_{1}=I\left(2 \pi ; \theta_{0}, I_{0}\right) .
$$

From the second equality of (3.2), we get that

$$
\frac{d I^{\frac{1}{2}}}{d t}=-\frac{1}{a \gamma} f\left(\gamma I^{\frac{1}{2}} S\left(\frac{\theta}{\omega}\right)\right) S\left(\frac{\theta}{\omega}\right)+\frac{1}{a \gamma} \varphi\left(t, \gamma I^{\frac{1}{2}} C\left(\frac{\theta}{\omega}\right)\right) S\left(\frac{\theta}{\omega}\right) .
$$

It follows from (3.3) that

$$
I(t)^{\frac{1}{2}}=I_{0}^{\frac{1}{2}}+O(1), \quad t \in[0,2 \pi], \quad I_{0} \rightarrow+\infty .
$$

Furthermore, we have that

$$
I(t)^{-\frac{1}{2}}=I_{0}^{-\frac{1}{2}}+O\left(I_{0}^{-1}\right), \quad t \in[0,2 \pi], \quad I_{0} \rightarrow+\infty .
$$

From (3.4) and the first equality of (3.2) we know that

$$
\frac{d \theta}{d t}=\omega+O\left(I_{0}^{-\frac{1}{2}}\right) .
$$

Consequently,

$$
\theta(t)=\theta_{0}+\omega t+O\left(I_{0}^{-\frac{1}{2}}\right), \quad t \in[0,2 \pi],
$$

which, together with (3.3), implies that

$$
\begin{aligned}
\frac{d I^{\frac{1}{2}}}{d t}= & -\frac{1}{a \gamma} f\left(\gamma I_{0}^{\frac{1}{2}} S\left(t+\frac{\theta_{0}}{\omega}\right)+O(1)\right) S\left(t+\frac{\theta_{0}}{\omega}\right) \\
& +\frac{1}{a \gamma} \varphi\left(t, \gamma I_{0}^{\frac{1}{2}} C\left(t+\frac{\theta_{0}}{\omega}\right)+O(1)\right) S\left(t+\frac{\theta_{0}}{\omega}\right)+O\left(I_{0}^{-\frac{1}{2}}\right) .
\end{aligned}
$$


By integrating (3.6) on $[0,2 \pi]$ we deduce that

$$
\begin{aligned}
I_{1}^{\frac{1}{2}}= & I_{0}^{\frac{1}{2}}-\frac{1}{a \gamma} \int_{0}^{2 \pi} f\left(\gamma I_{0}^{\frac{1}{2}} S\left(t+\frac{\theta_{0}}{\omega}\right)+O(1)\right) S\left(t+\frac{\theta_{0}}{\omega}\right) d t \\
& +\frac{1}{a \gamma} \int_{0}^{2 \pi} \varphi\left(t, \gamma I_{0}^{\frac{1}{2}} C\left(t+\frac{\theta_{0}}{\omega}\right)+O(1)\right) S\left(t+\frac{\theta_{0}}{\omega}\right) d t+O\left(I_{0}^{-\frac{1}{2}}\right) .
\end{aligned}
$$

Similarly, substituting (3.4) and (3.5) into the first equality of (3.2), we obtain that for all $t \in[0,2 \pi]$,

$$
\begin{aligned}
\frac{d \theta}{d t}= & \omega-\frac{\gamma}{2} I_{0}^{-\frac{1}{2}} f\left(\gamma I_{0}^{\frac{1}{2}} S\left(t+\frac{\theta_{0}}{\omega}\right)+O(1)\right) C\left(t+\frac{\theta_{0}}{\omega}\right) \\
& -\frac{\gamma}{2} I_{0}^{-\frac{1}{2}} \varphi\left(t, \gamma I_{0}^{\frac{1}{2}} C\left(t+\frac{\theta_{0}}{\omega}\right)+O(1)\right) C\left(t+\frac{\theta_{0}}{\omega}\right)+O\left(I_{0}^{-1}\right) .
\end{aligned}
$$

Therefore we have

$$
\begin{aligned}
\theta_{1}= & \theta_{0}+2 \omega \pi+\frac{\gamma}{2} I_{0}^{-\frac{1}{2}} \int_{0}^{2 \pi} f\left(\gamma I_{0}^{\frac{1}{2}} S\left(t+\frac{\theta_{0}}{\omega}\right)+O(1)\right) C\left(t+\frac{\theta_{0}}{\omega}\right) d t \\
& -\frac{\gamma}{2} I^{-\frac{1}{2}} \int_{0}^{2 \pi} \varphi\left(t, \gamma I_{0}^{\frac{1}{2}} C\left(t+\frac{\theta_{0}}{\omega}\right)+O(1)\right) C\left(t+\frac{\theta_{0}}{\omega}\right) d t+O\left(I_{0}^{-1}\right) .
\end{aligned}
$$

Set $r=I^{\frac{1}{2}} ;$ then we obtain

$$
\left\{\begin{aligned}
\theta_{1}= & \theta_{0}+2 \omega \pi+\frac{\gamma}{2} r_{0}^{-1} \int_{0}^{2 \pi} f\left(\gamma r_{0} S\left(t+\frac{\theta_{0}}{\omega}\right)+O(1)\right) C\left(t+\frac{\theta_{0}}{\omega}\right) d t \\
& -\frac{\gamma}{2} r_{0}^{-1} \int_{0}^{2 \pi} \varphi\left(t, \gamma r_{0} C\left(t+\frac{\theta_{0}}{\omega}\right)+O(1)\right) C\left(t+\frac{\theta_{0}}{\omega}\right) d t+O\left(r_{0}^{-2}\right), \\
r_{1}= & r_{0}-\frac{1}{a \gamma} \int_{0}^{2 \pi} f\left(\gamma r_{0} S\left(t+\frac{\theta_{0}}{\omega}\right)+O(1)\right) S\left(t+\frac{\theta_{0}}{\omega}\right) d t \\
& +\frac{1}{a \gamma} \int_{0}^{21} \varphi\left(t, \gamma r_{0} C\left(t+\frac{\theta_{0}}{\omega}\right)+O(1)\right) S\left(t+\frac{\theta_{0}}{\omega}\right) d t+O\left(r_{0}^{-1}\right) .
\end{aligned}\right.
$$

We define

$$
\begin{aligned}
& \psi_{1}\left(\theta_{0}, r_{0}\right)=\frac{\gamma}{2} \int_{0}^{2 \pi} f\left(\gamma r_{0} S\left(t+\frac{\theta_{0}}{\omega}\right)+O(1)\right) C\left(t+\frac{\theta_{0}}{\omega}\right) d t \\
& \psi_{2}\left(\theta_{0}, r_{0}\right)=-\frac{1}{a \gamma} \int_{0}^{2 \pi} f\left(\gamma r_{0} S\left(t+\frac{\theta_{0}}{\omega}\right)+O(1)\right) S\left(t+\frac{\theta_{0}}{\omega}\right) d t \\
& \psi_{3}\left(\theta_{0}\right)=\frac{\gamma}{2} \int_{0}^{2 \pi} \varphi\left(t, \gamma r_{0} C\left(t+\frac{\theta_{0}}{\omega}\right)+O(1)\right) C\left(t+\frac{\theta_{0}}{\omega}\right) d t \\
& \psi_{4}\left(\theta_{0}\right)=\frac{1}{a \gamma} \int_{0}^{2 \pi} \varphi\left(t, \gamma r_{0} C\left(t+\frac{\theta_{0}}{\omega}\right)+O(1)\right) S\left(t+\frac{\theta_{0}}{\omega}\right) d t
\end{aligned}
$$

Lemma 3.1. Assume that the limits $\lim _{x \rightarrow+\infty} f(x)=f(+\infty), \lim _{x \rightarrow-\infty} f(x)=f(-\infty)$ exist and are finite. Then, for $r_{0} \rightarrow+\infty$, we have

$$
\begin{aligned}
& \psi_{1}\left(\theta_{0}, r_{0}\right)=\frac{\gamma}{2}\left[f(+\infty) \int_{J_{3}} C\left(t+\frac{\theta_{0}}{\omega}\right) d t+f(-\infty) \int_{J_{4}} C\left(t+\frac{\theta_{0}}{\omega}\right) d t\right]+o(1), \\
& \psi_{2}\left(\theta_{0}, r_{0}\right)=-\frac{1}{a \gamma}\left[f(+\infty) \int_{J_{3}} S\left(t+\frac{\theta_{0}}{\omega}\right) d t+f(-\infty) \int_{J_{4}} S\left(t+\frac{\theta_{0}}{\omega}\right) d t\right]+o(1), \\
& \psi_{3}\left(\theta_{0}\right)=\frac{\gamma}{2}\left[\int_{J_{1}} \varphi(t,+\infty) C\left(t+\frac{\theta_{0}}{\omega}\right) d t+\int_{J_{2}} \varphi(t,-\infty) C\left(t+\frac{\theta_{0}}{\omega}\right) d t\right]+o(1),
\end{aligned}
$$


$\psi_{4}\left(\theta_{0}\right)=\frac{1}{a \gamma}\left[\int_{J_{1}} \varphi(t,+\infty) S\left(t+\frac{\theta_{0}}{\omega}\right) d t+\int_{J_{2}} \varphi(t,-\infty) S\left(t+\frac{\theta_{0}}{\omega}\right) d t\right]+o(1)$, where $J_{1}=\left\{t: t \in(0,2 \pi), C\left(t+\frac{\theta_{0}}{\omega}\right)>0\right\}, J_{2}=\left\{t: t \in(0,2 \pi), C\left(t+\frac{\theta_{0}}{\omega}\right)<0\right\}$, $J_{3}=\left\{t: t \in(0,2 \pi), S\left(t+\frac{\theta_{0}}{\omega}\right)>0\right\}, J_{4}=\left\{t: t \in(0,2 \pi), S\left(t+\frac{\theta_{0}}{\omega}\right)<0\right\}$.

Proof. We only give the proof of the second equality; the others are analogous. First of all, observe that the set of the zeros of $C\left(t+\frac{\theta_{0}}{\omega}\right)$ has measure zero; therefore we can write

$$
\begin{aligned}
\psi_{2}\left(\theta_{0}, r_{0}\right)= & -\frac{1}{a \gamma} \int_{0}^{2 \pi} f\left(\gamma r_{0} S\left(t+\frac{\theta_{0}}{\omega}\right)+O(1)\right) S\left(t+\frac{\theta_{0}}{\omega}\right) d t \\
= & -\frac{1}{a \gamma} \int_{J_{3}} f\left(\gamma r_{0} S\left(t+\frac{\theta_{0}}{\omega}\right)+O(1)\right) S\left(t+\frac{\theta_{0}}{\omega}\right) d t \\
& -\frac{1}{a \gamma} \int_{J_{4}} f\left(\gamma r_{0} S\left(t+\frac{\theta_{0}}{\omega}\right)+O(1)\right) S\left(t+\frac{\theta_{0}}{\omega}\right) d t
\end{aligned}
$$

By the boundedness of $f$, we can apply the Lebesgue dominate convergence theorem for $r_{0} \rightarrow+\infty$ to both the integrals on the right-hand side of above equality to obtain the results.

Lemma 3.2. $\int_{0}^{2 \pi} \psi_{4}\left(\theta_{0}\right) d \theta_{0}=0$.

Proof. Since $\psi_{4}\left(\theta_{0}\right)=\dot{\psi}_{3}\left(\theta_{0}\right), \psi_{4}\left(\theta_{0}\right)$ and $\psi_{3}\left(\theta_{0}\right)$ are $2 \pi$-periodic functions in $\theta_{0}$ (notice that $C\left(t+\frac{\theta_{0}}{\omega}\right)$ and $S\left(t+\frac{\theta_{0}}{\omega}\right)$ are $2 \pi$-periodic in $\left.\theta_{0}\right)$.

3.3. The proof of Theorem 1.1. Now we are in a position to give the proof of Theorem 1.1.

Consider the Poincaré mapping $P:\left(\theta_{0}, r_{0}\right) \rightarrow\left(\theta_{1}, r_{1}\right)$. From Lemma 3.1 we know that $P$ can be expressed in the form

$$
\left\{\begin{array}{l}
\theta_{1}=\theta_{0}+2 \alpha \pi+\frac{1}{r_{0}} \mu_{1}\left(\theta_{0}\right)+H\left(\theta_{0}, r_{0}\right) \\
r_{1}=r_{0}+\mu_{2}\left(\theta_{0}\right)+G\left(\theta_{0}, r_{0}\right)
\end{array}\right.
$$

where $H, G$ are continuous functions that satisfy

$$
H\left(\theta_{0}, r_{0}\right)=o\left(\frac{1}{r_{0}}\right), \quad G\left(\theta_{0}, r_{0}\right)=o(1) \quad \text { as } \quad r_{0} \rightarrow+\infty,
$$

and

$$
\mu_{1}\left(\theta_{0}\right)=\phi_{1}\left(\theta_{0}\right)-\phi_{3}\left(\theta_{0}\right), \quad \mu_{2}\left(\theta_{0}\right)=\phi_{2}\left(\theta_{0}\right)+\phi_{4}\left(\theta_{0}\right)
$$

with

$$
\begin{aligned}
& \phi_{1}\left(\theta_{0}\right)=\frac{\gamma}{2}\left[f(+\infty) \int_{J_{3}} C\left(t+\frac{\theta_{0}}{\omega}\right) d t+f(-\infty) \int_{J_{4}} C\left(t+\frac{\theta_{0}}{\omega}\right) d t\right] \\
& \phi_{2}\left(\theta_{0}\right)=-\frac{1}{a \gamma}\left[f(+\infty) \int_{J_{3}} S\left(t+\frac{\theta_{0}}{\omega}\right) d t+f(-\infty) \int_{J_{4}} S\left(t+\frac{\theta_{0}}{\omega}\right) d t\right], \\
& \phi_{3}\left(\theta_{0}\right)=\frac{\gamma}{2}\left[\int_{J_{1}} \varphi(t,+\infty) C\left(t+\frac{\theta_{0}}{\omega}\right) d t+\int_{J_{2}} \varphi(t,-\infty) C\left(t+\frac{\theta_{0}}{\omega}\right) d t\right], \\
& \phi_{4}\left(\theta_{0}\right)=\frac{1}{a \gamma}\left[\int_{J_{1}} \varphi(t,+\infty) S\left(t+\frac{\theta_{0}}{\omega}\right) d t+\int_{J_{2}} \varphi(t,-\infty) S\left(t+\frac{\theta_{0}}{\omega}\right) d t\right],
\end{aligned}
$$

where $J_{1}, J_{2}, J_{3}$, and $J_{4}$ are defined in Lemma 3.1. Clearly $\mu_{1}, \mu_{2}$ are Lipschitz continuous. On the other hand, it follows from $f(+\infty)<0<f(-\infty)$ that $\phi_{2}\left(\theta_{0}\right)>0$ 
for $\theta_{0} \in \mathbb{S}^{1}$. Therefore from Lemma 3.2 we get that

$$
\int_{0}^{2 \pi} \mu_{2}\left(\theta_{0}\right) d \theta_{0}=\int_{0}^{2 \pi} \phi_{2}\left(\theta_{0}\right) d \theta_{0}>0 .
$$

Applying Proposition 2.1 to the Poincaré mapping $P$, we then obtain the proof of first part of Theorem 1.1.

Since $\alpha$ is irrational and in view of the expression for the Poincare mapping (3.7), it follows that $\left(r_{1}, \theta_{1}\right)$ and $\left(r_{0}, \theta_{0}\right)$ never lie on the ray $\theta=\theta_{0}$ for $r_{0}$ large enough. So the Poincaré mapping (3.7) meets all the assumptions of the PoincaréBohl Theorem. Thus $P$ has at least one fixed point. Consequently Eq.(1.4) has at least one $2 \pi$-periodic solution. Therefore we have finished the proof of Theorem 1.1.

\section{REFERENCES}

[1] J. M. Alonso and R. Ortega, Roots of unity and unbounded motions of an asymmetric oscillator, J. Differential Equations 143(1998), 201-220. MR.1604908 (99a:34102)

[2] W. Dambrosio, A note on the existence of unbounded solutions to a perturbed asymmetric oscillator, Nonlinear Anal. 50(2002), 333-346. MR1906465 (2003d:34086)

[3] E. N. Dancer, Boundary value problems for weakly nonlinear ordinary differential equations, Bull. Aust. Math. Soc. 15(1976), 321-328. MR0430384 (55:3389)

[4] E. N. Dancer, On the Dirichlet problem for weakly nonlinear elliptic partial differential equations, Proc. Roy. Soc. Edinburgh 76A(1977), 283-300. MR.0499709 (58:17506)

[5] P. Drabek and S. Invernizzi, On the periodic boundary value theorem for forced Duffing equation with jumping nonlinearity, Nonlinear Anal. 10(1986), 643-650. MR0849954 (87j:34077)

[6] C. Fabry and A. Fonda, Nonlinear resonance in asymmetric oscillators, J.Differential Equations 147(1998), 58-78. MR.1632669 (99d:34070)

[7] C. Fabry and J. Mawhin, Oscillations of a forced asymmetric oscillator at resonance, Nonlinearity 13(2000), 1-13. MR 1758987 (2001d:34059)

[8] S. Fučik, Solvability of nonlinear equations and boundary value problems, Reidel, Dordrecht, 1980. MR0620638 (83c:47079)

[9] T. Gallouet and O. Kavian, Resonance for jumping nonlinearities, Comm. Partial Diff. Equations 7(1982), 325-342. MR0646710 (84f:35054)

[10] P. Habets, M. Ramos and L. Sanchez, Jumping nonlinearities for Neumann boundary value problems with positive forcing, Nonlinear Anal. 20(1993), 533-549. MR1207529 (94f:34050)

[11] M. Kunze, T. Küpper and B. Liu, Boundedness and unboundedness of solutions for reversible oscillators at resonance, Nonlinearity 14(2001), 1105-1122. MR.1862814 (2002g:34079)

[12] A. C. Lazer and P. J. McKenna, A semi-Fredholm principle for periodically forced systems with homogeneous nonlinearities, Proc. Amer. Math. Soc. 106(1989), 119-125. MR0942635 (89i:34056)

[13] A. C. Lazer and P. J. McKenna, Large-amplitude periodic oscillations in suspension bridge: some new connections with nonlinear analysis, SIAM. Rev.32(1990), 537-578. MR1084570 (92g:73059)

[14] X. Li and Q. Ma, Boundedness of solutions for second order differential equations with asymmetric nonlinearity, J. Math. Anal. Appl. 314(2006), 233-253. MR2183549 (2006h:34067)

[15] X. Li, Boundedness and unboundedness of solutions of reversible oscillators at resonance, Nonlinear Anal.65 (2006), 514-533. MR2231069

[16] B. Liu, Boundedness in asymmetric oscillations, J. Math. Anal. Appl 231(1999), 355-373. MR.1669195 (2000c:34093)

[17] R. Ortega, Asymmetric oscillators and twist mappings, J. London Math. Soc. 53(1996), 325-342. MR.1373064 (96k:34093)

[18] R. Ortega, Boundedness in a piecewise linear oscillator and a variant of the small twist theorem, Proc. London Math .Soc. 79(1999), 381-413. MR1702247 (2000g:34055)

[19] R. Ortega, Invariant curves of mappings with averaged small twist, Advanced Nonlinear Studies 1(2001), 14-39. MR.1850202 (2002h:37067) 
[20] Z. Wang, Irrational rotation numbers and unboundedness of solutions of the second order differential equations with asymmetric nonlinearities, Proc. Amer. Math. Soc.131 (2003), 523-531. MR.1933343 (2003g:37084)

School of Mathematical Sciences, Beijing Normal University, Beijing 100875, PeoPLE'S REPUBLiC OF CHINA

E-mail address: xli@bnu.edu.cn

School of Mathematical Sciences, Beijing Normal University, Beijing 100875, PeoPLE'S REPUBLiC OF CHINA 